\title{
Interaction of Particles in the Near Field and Opposition Effects in Regolith-Like Surfaces
}

\author{
E. V. Petrova ${ }^{a}$, V. P. Tishkovets ${ }^{b}$, and K. Jockers ${ }^{c}$ \\ ${ }^{a}$ Space Research Institute, Russian Academy of Sciences, Profsoyuznaya ul. 84/32, Moscow, 117997 Russia \\ ${ }^{b}$ Institute of Radioastronomy NASU, Chervonopraporna 4, Kharkiv, 61002 Ukraine \\ ${ }^{c}$ Max Planck Institute for Solar System Research, Katlenburg-Lindau, D-37191 Germany \\ Received June 24, 2008
}

\begin{abstract}
The explanation of the opposition effects observed in brightness and polarization in different celestial bodies and laboratory samples is still far from being complete. The shadow hiding and coherent backscattering mechanisms are mentioned most frequently in this connection. In the present work, we consider one more scattering mechanism - the interaction of particles in the near field - and its influence on the brightness and polarization of light scattered by ensembles of particles at small phase angles. First, we analyze two manifestations of this mechanism: the field inhomogeneity in the vicinity of the scatterers and the shielding of particles by each other at distances compared with their sizes. Then, we use the model regolith described as an ensemble of clusters as constituents and compare the contributions of the coherent backscattering and the near-field effect to the intensity and polarization of light when the porosity of the ensemble is varied. The modeling confirms that the phase dependences of the intensity and polarization of light scattered by complex structures in the backscattering domain is mainly caused by these two mechanisms. The coherent backscattering works more effectively in sparse media, while the near-field effect manifests itself in more compact ensembles of wavelength-sized particles. However, it is difficult to distinguish quantitatively their contributions, even in models of simple structures. A number of observations, especially of moderate- and low-albedo objects, can be explained only by invoking the near-field effect.
\end{abstract}

PACS: 42.25-p; 96.12K-; 96.25-f

DOI: $10.1134 / \mathrm{S} 0038094609020026$

\section{INTRODUCTION}

Solar electromagnetic radiation scattered by particles in the atmosphere or on the surface of celestial bodies provides us with a potentially rich source of information on the properties of the planets, their satellites, asteroids, and comets. However, some features in the phase functions of brightness and polarization, which were found long ago during observations of comets and atmosphereless celestial bodies, have not been unambiguously explained yet. They are the socalled opposition effect in brightness (a nonlinear increase of brightness observed at decreasing phase angles near opposition) and the negative branch of polarization (a change of the linear polarization sign from positive to negative at phase angles less than $\approx 20^{\circ}$ ). The data of observations, both photometric and polarimetric, are widely presented in the literature (see, e.g., Lyot, 1929; Dollfus and Bowell, 1971; Dollfus and Auriere, 1974; Leinert, 1975; Zellner and Gradie, 1976; Zellner et al., 1977; Dollfus and Geake, 1977; Kuga and Ishumaru, 1984; Dollfus, 1989; Shkuratov et al., 2002; Jockers, 1999; Belskaya and Shevchenko, 2000; Kiselev et al., 2002; Rosenbush et al., 2002; Boeh- nhardt et al., 2004; Shkuratov et al., 2004; Rosenbush et al., 2006). In many cases, but not always (e.g., not for F-type asteroids (Belskaya et al., 2005)), the opposition effects in brightness and polarization accompany each other.

Among the mechanisms proposed for explaining the phase curves of brightness and polarization of atmosphereless celestial bodies, the following ones can be distinguished.

To explain the backscattering enhancement of brightness, the shadowing models (Hapke, 1986; Lumme and Bowell, 1981) were used first. They are based on geometric optics and attribute this effect to the elimination of mutual shadows cast by individual regolith grains upon each other as the phase angle draws closer towards zero. However, this explanation fails to describe a very narrow brightening (less than $1^{\circ}$ ) observed for many high-albedo objects, since in this case it would imply an unrealistically low volume density of the upper regolith layer.

The features in the phase curves of brightness and polarization observed in particulate low-reflectivity 
media can be partly the remnants of the details of the scattering phase curves of individual particles dampened by multiple scattering in the medium. Laboratory measurements of the scattering on the independent particles of complex structure and on the samples composed of these particles confirmed this thesis on the whole (Shkuratov et al., 2004; 2006). However, some features in the behavior of the phase functions do not satisfy this simple scheme. Moreover, in this case, the cause of the opposition effects demonstrated by individual particles of a complex structure remains obscure. Recently, this problem has been actively discussed in publications; among the recent publications see, for example, the papers by Shkuratov et al. (2004), Tishkovets et al. (2002a; 2002b; 2004a), Muinonen (2004), and Petrova et al. (2004).

Among the mechanisms proposed to explain the phase curves of the polarization of atmosphereless celestial bodies (see, e.g., the review by Shkuratov et al., 1994), the coherent backscattering mechanism (also known as weak photon localization) is most widely accepted. It induces a sharp increase of brightness near opposition by the constructive interference of waves scattered multiply inside the medium along direct and reverse trajectories (e.g., van Albada and Lagendijk, 1985; Wolf and Maret, 1985; Akkermans et al., 1986; Barabanenkov et al., 1991). The same mechanism was proposed by Shkuratov (1989) and Muinonen (1990) to explain the negative polarization branch observed for many atmosphereless bodies of the Solar System at phase angles smaller than $25^{\circ}$. Later, Mishchenko (1993) showed that the coherent backscattering may cause only a narrow minimum of negative polarization that sometimes is observed at very small phase angles (see also Mishchenko et al., 2006a).

However, in some cases the observed behavior of the phase curves of polarization near opposition cannot be explained only by the coherent backscattering. Another mechanism that can produce substantial influence on the intensity and the polarization of light scattered by complex ensembles is the near-field effect. (In publications, the near field is associated with a field, the amplitude of which decreases faster than $1 / r$, where $r$ is the distance to the scatterer (Greffet and Carminati, 1998)) It is mostly noticeable in closely packed media, since its action is based on the peculiarities of the scattering of the near field when the distances between the scatterers are comparable to their sizes. Although the properties of the near-field effect have been already been substantially considered in publications (see, e.g., Tishkovets, 1998; Tishkovets and Litvinov, 1999; Tishkovets et al., 1999; 2004a; 2004b), our recent paper (Petrova et al., 2007), where we discussed the manifestation of two mechanisms - the coherent backscattering and the near-field effect-in the scattering near opposition, faced keen criticism (Shkuratov and Zubko, 2008). Our opponents doubt the effectiveness of the scattering mechanism based on the interaction of particles in the near field. The issue of the paper by Shkuratov and Zubko (2008) shows that the problem on the contribution of the near-field effect to the intensity and the polarization of light scattered by closely packed media should be specially considered in more detail. Since in our reply to the opponents we were limited in volume (Petrova et al., 2008), we set this purpose in the present paper that, consequently, has taken the review traits.

In this paper, we analyze the influence of the near field on the characteristics of light scattered by closely packed ensembles of particles. We can consider only a limited number of particles in these systems, since the present day theory of light scattering by media ignores the interaction of scatterers in the near field. In our calculations, we used the model of spherical particles composing the ensemble. This is not of principal importance for our results, although it makes the computational procedure much easier. In the first section, we present the main relations for describing light scattering by ensembles of spherical particles. With these relations, the characteristics of light scattered by ensembles of particles can be calculated with "turning on" and "turning off" the near field. In the next section, we explain the mechanism of the influence of the near field - the inhomogeneities of the field in the vicinity of the particles and the mutual shieldin-on the intensity and the polarization of the scattered light. We will call the effect connected with the peculiarities of the light scattering by particles in the near field as the near-field effect. Then, on the examples of clusters of spherical particles, we quantitatively analyze the contribution of the near field. In the next section, we qualitatively compare the contributions of the coherent backscattering and the near-field effect to the characteristics of light scattered near the opposition in dependence on the packing density of the scattering ensemble. The present modeling has allowed the areas of the strongest influence of each of the mechanisms to be found; it has been determined that the near-field effect can play a substantial part in the light scattering by moderate- and lowalbedo bodies composed of closely packed particles.

\section{DESCRIPTION OF THE LIGHT SCATTERING BY AN ENSEMBLE OF PARTICLES BEING IN THE FAR AND NEAR FIELD}

The characteristics of light scattered by an arbitrary particle or a system (cluster) of particles in the far zone are determined by the scattering matrix $\mathbf{F}$ that describes the 


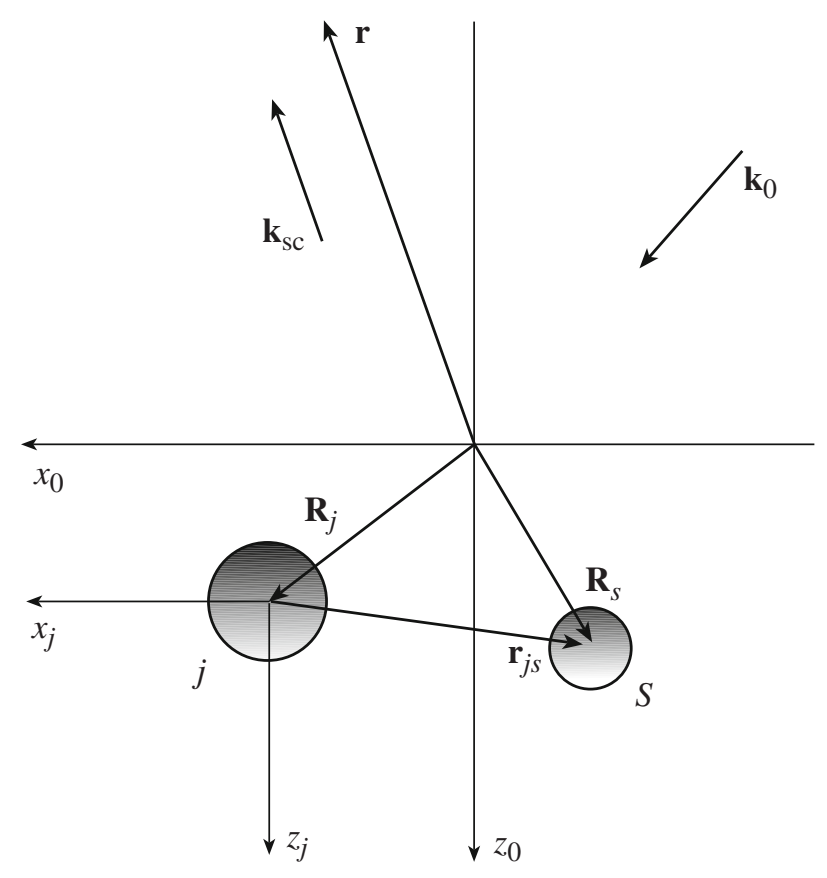

Fig. 1. The coordinate system for describing the light scattering by a cluster. See the text for notations.

transformation of the Stokes vector of the incident light $\mathbf{I}_{0}$ to that of the scattered light I (Mishchenko et al., 2002).

$$
\mathbf{I}=\frac{1}{\left(k_{0} r\right)^{2}} \mathbf{F I}_{0}
$$

Here, $k_{0}=2 \pi / \lambda, \lambda$ is the wavelength of the incident light, and $r$ is the distance from the center of a particle or a cluster to the observational point. The formulas for the elements of matrix $\mathbf{F}$ can be written in terms of the elements of the amplitude scattering matrix $\mathbf{S}$ (Mishchenko et al., 2002). Specifically, the matrix elements $F_{11}$ and $F_{21}$ are

$$
F_{11}=\sum_{p n}\left|S_{p n}\right|^{2}, \quad F_{21}=-\sum_{p n} S_{p n} S_{-p n}^{*}
$$

where $S_{p n}$ is the amplitude matrix in the so-called CP representation (in the circular polarization basis), $n$, and $p= \pm 1$ (see, e.g., Tishkovets and Jockers, 2006). When the nonpolarized light is scattered on randomly oriented particles or clusters, these elements determine the relative intensity $I$ and the degree of linear polarization $P$ of the scattered light

$$
I=\left\langle F_{11}\right\rangle, \quad P=-\left\langle F_{21}\right\rangle / I,
$$

where the angular brackets denote averaging over orientations of the particle or cluster.
Consider the scattering of a plane wave by a cluster composed of $N$ spherical homogeneous isotropic particles of arbitrary sizes and refractive indexes. Its amplitude matrix $S_{p n}$ can be presented as a sum of amplitude matrixes of all its particles (Mishchenko et al., 2002). The amplitude matrix $t_{p n}^{(j)}\left(\mathbf{k}_{0}, \mathbf{k}_{\mathrm{sc}}\right) j$ of the jth particle of the cluster $(j=1 \ldots N)$ has the following form (see, e.g., Tishkovets and Mishchenko, 2004; Tishkovets and Jockers, 2006; Tishkovets, 2007):

$$
\begin{gathered}
t_{p n}^{(j)}\left(\mathbf{k}_{0}, \mathbf{k}_{\mathrm{sc}}\right) \\
=\exp \left(-i \mathbf{k}_{\mathrm{sc}} \mathbf{R}_{j}\right) \sum_{L M} \frac{2 L+1}{2} A_{L M}^{(j p n)} D_{M p}^{* L}(\varphi, \vartheta, 0) .
\end{gathered}
$$

Here, $\mathbf{k}_{0}$ and $\mathbf{k}_{\mathrm{sc}}$ are the wave vectors of the incident and scattered waves, respectively $\left(k_{\mathrm{sc}}=k_{0}\right), \mathbf{R}_{j}$ is the radiusvector of the jth particle in the laboratory coordinate system $\left(x_{0}, y_{0}, z_{0}\right)$ with the origin in the center of the cluster (see Fig. 1), $D_{M p}^{L}(\varphi, \vartheta, 0)=\exp (-i M \varphi) d_{M p}^{L}(\vartheta)$ is the Wigner function (Varshalovich et al., 1988), and $\varphi$ and $\vartheta$ are the spherical angles of the vector $\mathbf{k}_{\mathrm{sc}}$ in the $\left(x_{0}, y_{0}, z_{0}\right)$ coordinate system. Index $L$ is in the interval $1 \leq L \leq L_{\max }$, where the maximal value $L_{\max }$ is determined by the particle's radius (Mishchenko et al., 2002), and index $M$ takes the values in the range of $-L \leq M \leq L$.

The coefficients $A_{L M}^{(j p n)}$ are determined from the system of equations (see, e.g., Tishkovets and Mishchenko, 2004; Tishkovets and Jockers, 2006; Tishkovets, 2007)

$$
\begin{gathered}
A_{L M}^{(j p n)}=a_{L}^{(j p n)} \exp \left(i \mathbf{k}_{0} \mathbf{R}_{j}\right) D_{M n}^{L}\left(\varphi_{0}, \vartheta_{0}, 0\right) \\
+\sum_{q} a_{L}^{(j p q)} \sum_{s=1}^{N} \sum A_{l m}^{(s q n)} H_{L M l m}^{(q)}\left(\mathbf{r}_{j s}\right),
\end{gathered}
$$

where $q= \pm 1, a_{L}^{(j p n)}=a_{L}^{(j)}+p n b_{L}^{(j)}, a_{L}^{(j)}$ and $b_{L}^{(j)}$ are the Mie coefficients for the jth particle (Mishchenko et al., 2002), $\varphi_{0}$ and $\vartheta_{0}$ are the spherical angles of the vector $\mathbf{k}_{0}$ in the $\left(x_{0}, y_{0}, z_{0}\right)$ coordinate system, and $\mathbf{r}_{j s}$ is the radius vector of the sth particle in the coordinate system of the jth particle. The origins of the coordinate systems connected with the particles are in the center of these particles, and their axes are parallel to those of the laboratory system (see Fig. 1). The coefficients of the addition theorem for the vector Helmholtz harmonics can 
be defined as follows (see, e.g., Tishkovets and Jockers, $\quad$ 2006; Tishkovets, 2007):

$$
H_{L M l m}^{(q)}\left(\mathbf{r}_{j s}\right)=\frac{2 l+1}{2}(-1)^{m} \sum_{l_{1}} i^{-l_{1}} h_{l_{1}}\left(k_{0} r_{j s}\right) D_{m_{1} 0}^{l_{1}}\left(\varphi_{j s}, \vartheta_{j s}, 0\right) C_{L M l-m}^{l_{1} m_{1}} C_{L q l-q}^{l_{1} 0}
$$

Here, $h_{l}\left(k_{0} r\right)$ is the Hankel spherical function, $\varphi_{j s}$ and $\vartheta_{j s}$ are the spherical angles of the vector $\mathbf{r}_{j s}$ in the $\left(x_{j}, y_{j}, z_{j}\right)$ coordinate system, the symbols indexed with $C$ are the Clebsch-Gordan coefficients (Varshalovich et al., 1988), $m_{1}=M-m$, and the index $l_{1}$ takes the values $|L-l| \leq l_{1} \leq L+l$.

Equations (4) and (5) describe the light scattering by an arbitrary cluster of spherical particles. In the right part of eq. (5), the first and second terms correspond to the scattering by the jth particle of the incident wave and of the other waves coming from all the other clusters' particles, respectively. This form of the system of equations (5) allows the coefficients $A_{L M}^{(j p n)}$ to be expanded in a series by scattering orders: the first term describes the single-scattering contribution; its substitution to the second term instead of $A_{l m}^{(s q n)}$ produces the double-scattering contribution, and so forth.

The coefficients (6) in the system of equations (5) completely describe the properties of the complex field between the particles of the cluster. When the particles are far from each other, in other words, they are in the far zones of each other, the waves propagating between the particles of the ensemble become spherical. To show this, we present the Hankel spherical function as a finite series (Gradshtain and Ryzhik, 1980)

$$
\begin{gathered}
h_{L}(x)=\sqrt{\frac{\pi}{2 x}} H_{L+1 / 2}^{(1)}(x) \\
=(-i)^{L+1} \frac{\exp (i x)}{x} \sum_{k=0}^{L}(-1)^{k} \frac{(L+k) !}{k !(L-k) !} \frac{1}{(2 i x)^{k}},
\end{gathered}
$$

where $H_{L+1 / 2}^{(1)}$ is the Hankel cylindrical function of a half-integer index. If $x \gg 1, x \gg L$, and $2 x \gg L^{2}$, only the term with $k=0$ can be taken into account in (7). In terms of the Hankel spherical function in (6), these inequations are equivalent to the assumption that the distances between the particles are much larger than the wavelength and their sizes, i.e., $r_{j s} \gg \lambda, r_{j s} \gg \tilde{a}_{j}+\tilde{a}_{s}$, and $2 r_{j s} \gg k_{0}\left(\tilde{a}_{j}+\tilde{a}_{s}\right)^{2}$, where $\tilde{a}_{j}$ and $\tilde{a}_{s}$ are the radii of the jth and sth particles. In this case, the coefficients (6) take the form

$$
\begin{gathered}
H_{L M l m}^{(q)}\left(r_{j s}\right) \\
=\frac{2 l+1}{2} \frac{\exp \left(i k_{0} r_{j s}\right)}{-i k_{0} r_{j s}} D_{M q}^{L}\left(\varphi_{s j}, \vartheta_{s j}, 0\right) D_{m q}^{*^{l}}\left(\varphi_{s j}, \vartheta_{s j}, 0\right)
\end{gathered}
$$

where $\varphi_{s j}$ and $\vartheta_{s j}$ are the spherical angles of the vector $r_{s j}$ in the coordinate system of the sth particle (Tishkovets and Mishchenko, 2004).

The coefficients (8) correspond to the spherical wave propagating from the $s$ th particle to the jth one. The amplitude of this wave depends on the direction of the $\mathbf{r}_{s j}$ vector and decreases as $r_{s j}^{-1}$. Opposite to the coefficients (8), the coefficients (6) contain the terms decreasing faster than $r_{s j}^{-1}$. In publications, such terms are attributed to the near field (Greffet and Carminati, 1998). Thus, if the near-field components are ignored in (6), we come to the coefficients (8) describing the spherical waves, though the character of the real field between closely located particles is complex. In this simplification, we ignore both the inhomogeneity of waves between the particles and the shielding of particles by each other, which is unavoidable, when $r_{j s} \approx \tilde{a}_{j}+\tilde{a}_{s}$.

It is necessary to note that the presently existing theories and models of the multiple scattering of light by disperse media are based on the concept that the secondary waves propagating between the scatterers in the medium are spherical. Specifically, the classical radiative transfer equation ignores the near field (Mishchenko et al., 2006). In models of the negative polarization based on the interference mechanism, it is also assumed that the spherical waves propagate between the scatterers (Shkuratov, 1989; Muinonen, 1990; 2004; Mishchenko, 2008).

In the next section, we will qualitatively consider the influence of the near field on the characteristics of light scattered by ensembles of particles.

\section{THE WAY THE NEAR FIELD AFFECTS THE SCATTERING CHARACTERISTICS}

In order to explain the way the near-field components affect the characteristics of the scattered light, it is necessary to consider the field structure in the vicinity of the particles. If the scattered wave contained only the components decreasing as $1 / r$, the wave leaving the particle would be spherical far from the scatterer and close to it as well. Then, in the close vicinity of the scatterer, the propagation directions of the scattered and incident waves would not coincide (expect the direction of $\mathbf{k}_{\mathrm{sc}}=\mathbf{k}_{0}$ ), and these waves would not be connected. However, since there are components decreasing faster than $1 / r$ in the scattered wave, the incident and the scattered fields are connected at some distance from the 


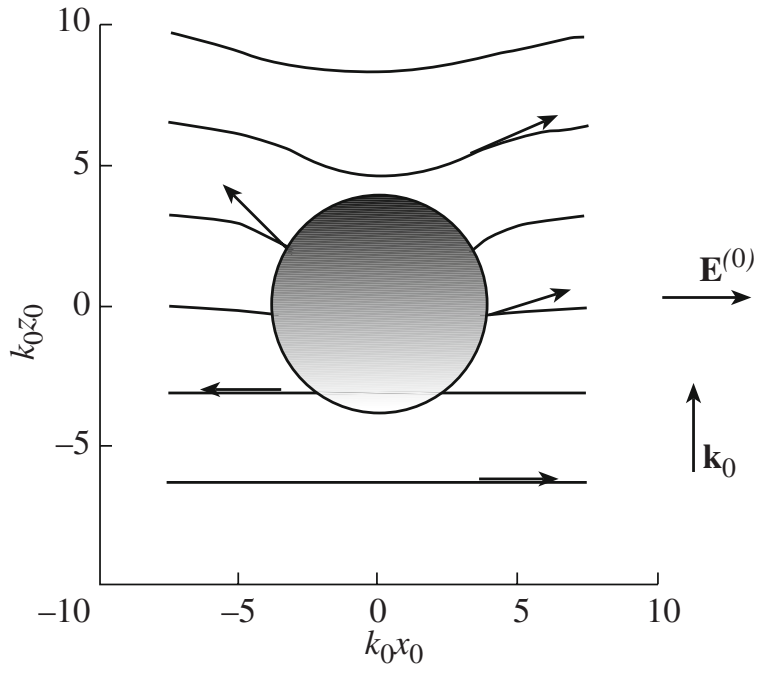

Fig. 2. The scheme shows the constant-phase surfaces and directions of electric field vectors (sum of the incident and scattered waves) in the close vicinity of a particle with a size parameter $X=k_{0} \tilde{a}=4.0$ and a refractive index $m=1.32+$ $0.05 i$. The incident wave propagates along the wave vector $\mathbf{k}_{0}$ and is polarized in the $x_{0} z_{0}$ plane. The thick lines indicate the constant-phase surface at a distance $k_{0} z_{0} \approx \pi$ from the surface $k_{0} z_{0}=0$ (the particle's center).

scatterer. This connection between the incident and scattered fields in the particle's vicinity leads to the inhomogeneity of the total field (a sum of the incident and scattered fields) in this area. This effect is mostly expressed if the scatterer is comparable in size to the wavelength. Direct calculations using the Lorentz-Mie theory for spherical particles show that the constant- phase surface of the total field is funnel shaped in the particle vicinity (Fig. 2). Consequently, the field inhomogeneity near the particle causes the turn of the total field vector relative to the incident field vector. As a result of this turn, the component of the total field $E_{z} \neq 0$ (see also Tishkovets, 1998; Tishkovets and Litvinov, 1999; Tishkovets et al., 1999; 2004a; Petrova et al., 2007).

Under such conditions, neighboring particles experience the influence of the inhomogeneous field and, consequently, scatter light in a different way than predicted by the theory considering only plane waves. To examine the influence of the field inhomogeneity in the vicinity of particles on the characteristics of light scattered by the ensemble of particles in more detail, let us consider Rayleigh test particles placed on the constantphase surface marked by thick lines in Fig. 2. This surface is distant from the surface of $k_{0} z_{0}=0$ by $k_{0} z_{0} \approx \pi$. Figure 3 shows the scheme illustrating the conditions of the light scattering by four test particles located in the homogeneous field (a) and in the inhomogeneous zone near the larger particle (b).

Let us first investigate the case when the incident field is polarized in the scattering plane (as shown in Fig. 3). If the test particles are far from each other and from other particles, i.e., they experience a homogeneous field (Fig. 3a), their dipole moments are parallel to the $x_{0}$ axis. In this case, the intensity of the scattered light is determined by all four test particles-dipoles in the direction $\alpha=0^{\circ}$ and $180^{\circ}$, and it is zero in the direction $\alpha=90^{\circ}$. If the test particles are, however, in the inhomogeneous zone near a wavelength-sized particle (Fig. 3b), the dipole moments induced in particles 1 and 3 (a)

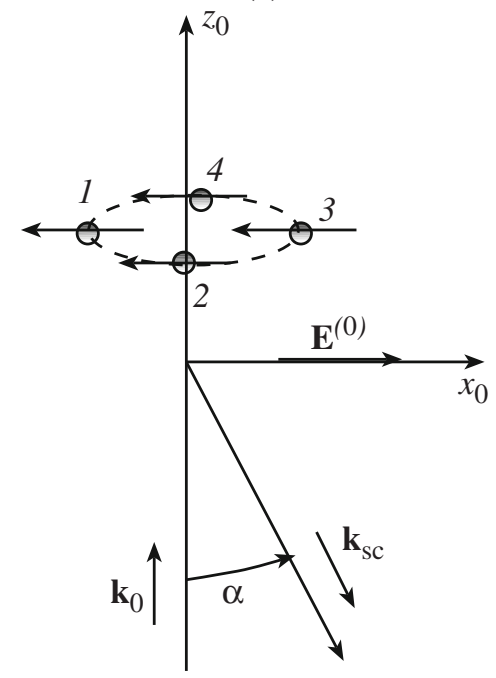

(b)

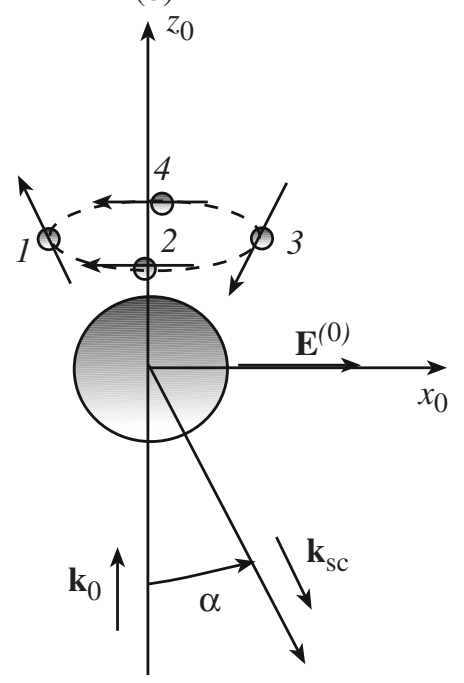

Fig. 3. The scheme for the scattering of homogeneous (a) and inhomogeneous (b) waves by the Rayleigh test particles. Particles 1 and 3 are in the $x_{0} z_{0}$ plane and particles 2 and 4 are in the $y_{0} z_{0}$ plane. The incident wave $\mathbf{E}^{(0)}$ propagates along the $z_{0}$ axis of a coordinate system (along the $\mathbf{k}_{0}$ vector) and is polarized in the $x_{0} z_{0}$ plane. The scattered wave propagates to the direction of the phase angle $\alpha$ (along the $\mathbf{k}_{\mathrm{sc}}$ vector). The vectors at the Rayleigh particles show the directions of the dipole moments induced. 
(a)

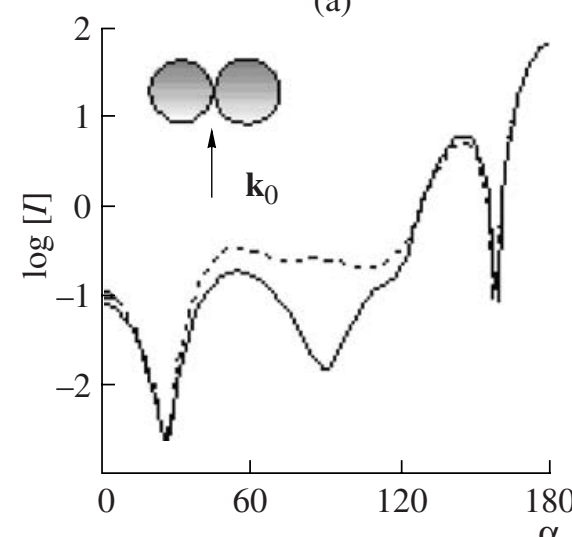

(b)

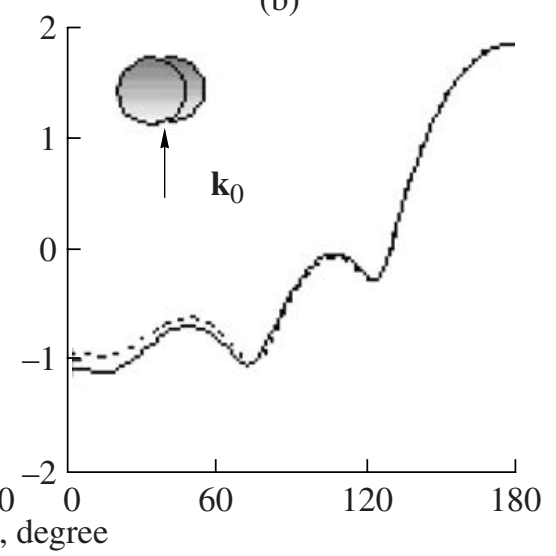

Fig. 4. The phase dependence of the intensity of light scattered by a bisphere, the axis of which is in the scattering plane (a) and in the perpendicular plane (b). The solid and dashed curves correspond to the calculations with the coefficients (6) (the near field is accounted for) and (8) (the near field is ignored), respectively. The calculated values of the relative intensity are normalized in the same way.

have a nonzero component in the direction of wave propagation, i.e., along the $z_{0}$ axis. This results in a decreasing of the intensity of the scattered light in the direction $\alpha=0^{\circ}$ and $180^{\circ}$, and the intensity in the direction $\alpha=90^{\circ}$ becomes nonzero. In both cases, the scattered wave is polarized in the same way as the incident one in the scattering plane.

When the incident wave is polarized perpendicular to the scattering plane, in the case of the homogeneous field, the scattered radiation is also polarized perpendicular to the scattering plane and the intensity does not depend on a phase angle. In the inhomogeneous field, particles 1 and 3 produce radiation that is polarized perpendicular to the scattering plane and does not depend on a phase angle. While the radiation scattered by particles 2 and 4 has a component parallel to the $z_{0}$ axis (i.e., polarized in the scattering plane) that depends on $\alpha$, the intensity decreases in the direction $\alpha=0^{\circ}$ and $180^{\circ}$ and increases in side directions.

So, at any polarization of the incident wave, the field inhomogeneity in the vicinity of the scattering particle induces the turn of the field vector and leads to the appearance of the component of the total field $E_{z} \neq 0$, which, in turn, diminishes the intensity of the light and causes negative polarization (see also Tishkovets, 1998; Tishkovets and Litvinov, 1999; Tishkovets et al., 1999; 2004a; Petrova et al., 2007). This effect should depend on the sizes of the particles and be mostly noticeable for particles comparable in size with the wavelength, since the field inhomogeneities near the scatterer are of a wavelength scale (Fig. 2).

One more manifestation of the interaction of particles in the near field is the shielding of particles by each other (Tishkovets, 2008). The scheme with the test dipoles (Fig. 3) can help to estimate qualitatively, for example, the result of the shielding of dipole 1 by particle 5 at the specified direction of observation. For the sake of simplicity, let us assume that at the specified polarization of the incident radiation, the dipole moment of particle 1 is oriented exactly opposite to the $\mathbf{k}_{\mathrm{sc}}$ vector. In this case, particle 1 does not radiate in the $\mathbf{k}_{\mathrm{sc}}$ direction. It is of no importance whether we take the shielding into account or not. When the incident radiation is polarized in the $y_{0} z_{0}$ plane, particle 1 would radiate like particle 3 does (or like all the particles in the homogeneous field) if the shielding is ignored. However, when particle 1 shields particle 5 , the latter does not radiate in this direction (i.e., does not contribute to the positive polarization). In other words, the shielding diminishes the contribution of the positively polarized scattered radiation and diminishes the intensity in the $\alpha$ direction. However, in the backscattering direction, dipole 1 contributes to the scattered radiation, which induces an increase in the intensity with respect to that in the $\alpha$ direction. (In this connection, we note that the geometric optics description of the shadowing effect leads to no negative polarization (Shkuratov et al., 1994; 2002; Shkuratov and Grynko, 2005) Contrary to the field inhomogeneity in the near zone, which is most noticeable for the wavelength-sized particles, the mutual shielding effect is independent of the sizes of the particles being in the near field.

In the next section, we consider the influence of the near field on the intensity and degree of linear polarization of light scattered by clusters of spherical particles. In our calculations, we use the formulas presented in the section "Description of the Light Scattering by an Ensemble of Particles Being in the Far and Near Field" and the coefficients $H_{L m l m}^{(q)}\left(\mathbf{r}_{j s}\right)$ in the form (6) or (8), 


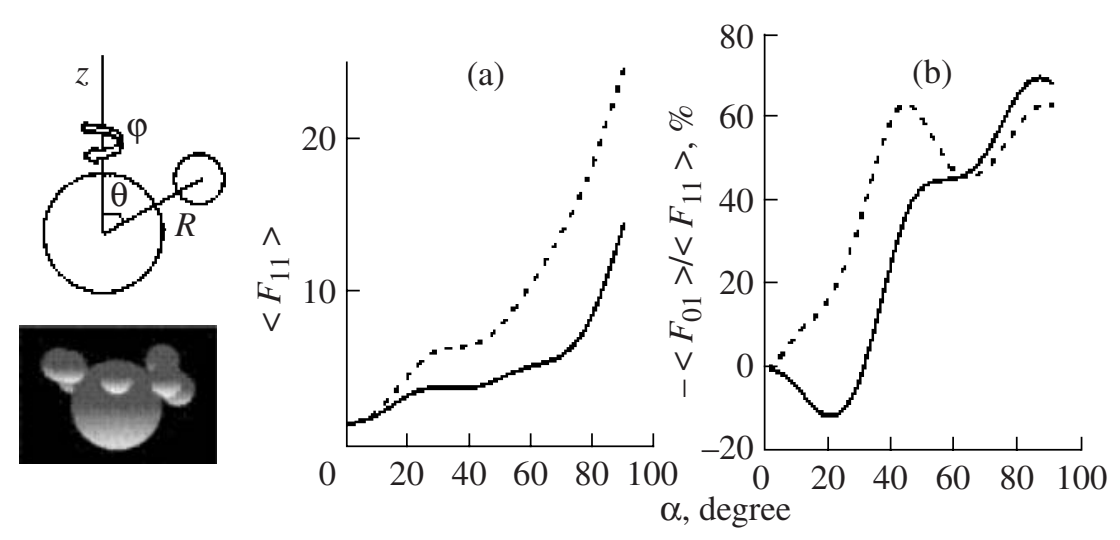

Fig. 5. The phase dependence of intensity (a) and linear polarization (b) of light scattered by the cluster shown as an insert leftward. The coordinates of eight small particles in the spherical system centered at the large particle (see the scheme on the left) are $R_{i}=5.5$, 6.5, 5.8, 6.7, 6.2, 5.9, 7.1, 6.8 (in units of size parameter), $\theta_{i}=75^{\circ}, 70^{\circ}, 65^{\circ}, 60^{\circ}, 55^{\circ}, 65^{\circ}, 75^{\circ}, 55^{\circ}$ and $\varphi_{i}=0^{\circ}, 45^{\circ}, 90^{\circ}, 135^{\circ}$, $180^{\circ}, 225^{\circ}, 270^{\circ}, 315^{\circ}$. The models calculated with the near-field components accounted for and ignored are shown with solid and dashed curves, respectively.

accounting for or ignoring the near-field components, respectively.

\section{THE NEAR-FIELD EFFECT IN CLUSTERS OF SPHERES}

It is convenient to consider the manifestation of the mutual shielding of particles comparable to the wavelength in size with an example of the light scattering by bispheres with components in contact. Figure 4 presents the intensity of light scattered by such bispheres orientated in two ways relative to the scattering plane coinciding with the plot plane. The size parameters of the bisphere's particles are $X=4$ and the refractive indexes are $m=1.32+i 0.05$. The incident plane wave is not polarized and its propagation direction is indicated with the $\mathbf{k}_{0}$ vector in the scheme.

The comparison of the models presented in Fig. 4 shows that, when the particles of the bisphere are in the scattering plane, the intensity of the scattered light in the direction of the bisphere's axis $\left(\alpha \approx 90^{\circ}\right)$ calculated with the near field taken into account is substantially lower (approximately by one order of magnitude) than that calculated with the near field ignored. However, if the bisphere is oriented perpendicular to the scattering plane, there is no difference between the models of the intensity of light scattered in side directions. Hence, in the case of the bisphere orientation in the scattering plane, the cause of the intensity decrease is in the mutual shielding (shadowing) of particles; in other words, each of the spheres is an obstacle for propagating the radiation scattered along the bisphere's axis. As has been noted in the section "Description of the Light Scattering by an Ensemble of Particles Being in the Far and Near Field," the coefficients (6) completely describe all the peculiarities in the field surrounding the particles, which are specifically realized in the mutual shielding of particles (Tishkovets, 2008). In fact, if the field components decreasing faster than $1 / r$ are ignored in the coefficients (6), these coefficients describe the spherical waves; saying this differently, in the field between the particles, the sizes of particles are ignored in comparison with the distances between them. In other words, in the approximation (8) the shielding concept itself loses its sense. Therefore, for the scattering direction parallel to the bisphere's axis, the intensity calculated within this approximation is much larger than that calculated with the interaction in the near field accounted for. The calculations fulfilled for the particles with the specified properties show that the shielding effect is noticeable up to the interparticle distances of an order of several of their diameters.

The influence of the near field on the intensity and the degree of linear polarization of the scattered light can be illustrated with a more complex example as well. Figure 5 displays the scattering characteristics of the partly oriented cluster (shown as an insert in the plot) consisting of one relatively large particle (the size parameter is $X=4.0$ and the refractive index is $m=1.32+i 0.05)$ and eight small particles (the size parameter is $X=1.5$ and the refractive index is $m=1.5+i 0.1$ ). The coordinates of the cluster's particles are provided in the figure heading. The incident light is from the bottom, along the $z$ axis. The characteristics of the cluster are averaged over a rotation of the cluster around the $z$ axis and by variations in the angles $\vartheta_{i}$ of small particles in interval $\pm 5^{\circ}$. The relatively high absorption and the random positioning of particles in the cluster were chosen in order to diminish the contribution of the wave interference which resulted from the regular arrangement of the particles.

The comparison of the models calculated with the near-field components accounted for and ignored show 


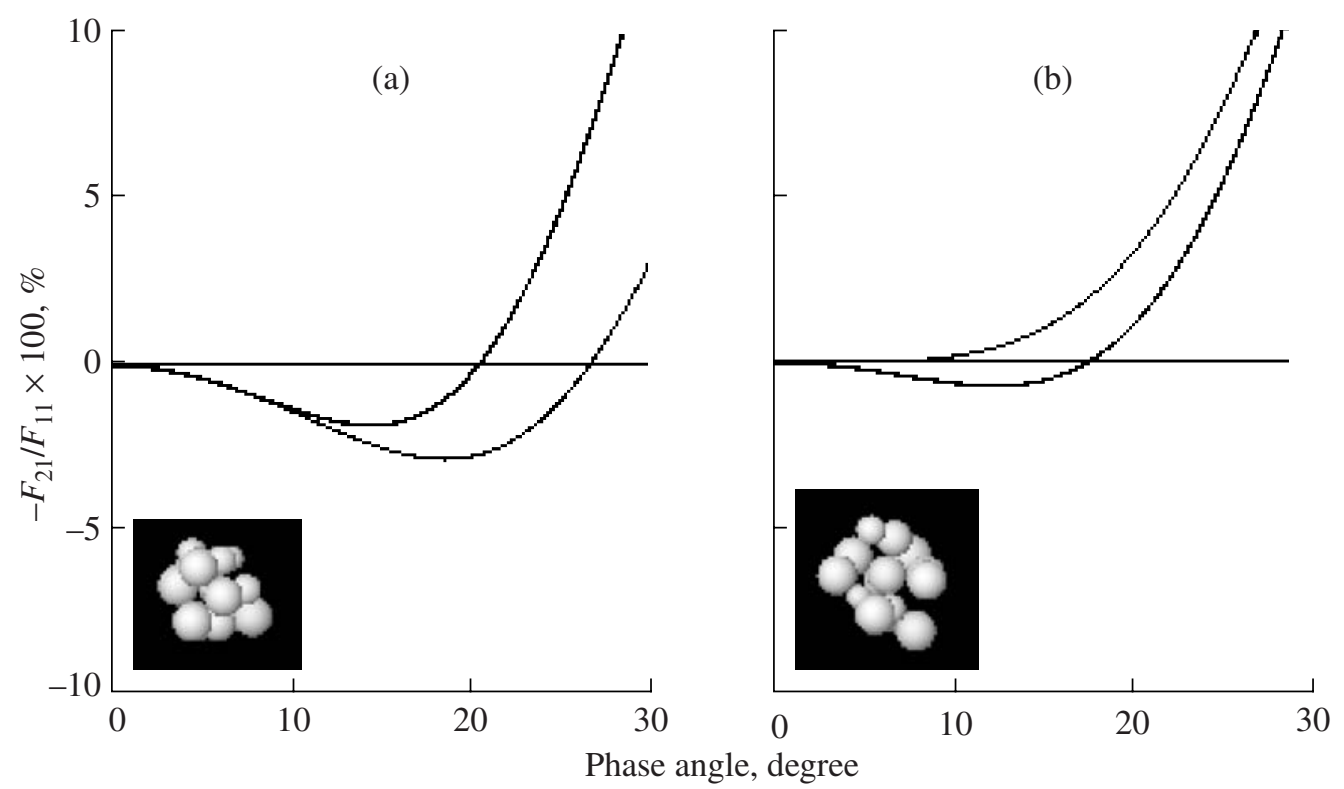

Fig. 6. The phase dependence of the degree of linear polarization for randomly oriented clusters with the porosity 0.52 (a) and 0.61 (b) calculated with the near-field components accounted for (thick lines) and ignored (thin lines). The refractive index of particles is $m=1.65+0.001 i$.

that the interaction of particles in the near field suppresses the intensity in a wide angular range and induces the negative polarization in the backscattering domain. It is worth noting that the intensity, at least for the given cluster, is mostly affected by shielding, since it is the shielding that suppresses the intensity in the side scattering directions. The influence of the shielding and the near-field inhomogeneity on the polarization is difficult to distinguish, because in this case both effects have the same sign.

\section{OPPOSITION PHENOMENA IN ENSEMBLES OF CLUSTERS}

As has been already mentioned in the Introduction, in the phase-angle range close to opposition, the behavior of the scattering characteristics of complex ensembles of particles is determined not only by the interaction of particles in the near field, but also by the coherent backscattering. Since the contribution of both mechanisms to the scattered radiation should be naturally determined by the properties of the particles and their packing density, the estimate of their influence on the intensity and polarization of light scattered by different objects is of practical importance in the interpretation of measurements. Consequently, the efficiencies of these mechanisms are required to be compared in dependence on the properties of the medium.

However, it is difficult to analyze the near-field contribution in the backscattering domain with the abovedescribed method directly isolating the near-field components, since the interaction of particles in the near field diminishes the multiple scattering contribution by means of shielding, rather than only inducing, the opposition phenomena. Hence, in the models ignoring the near-field components, i.e., ignoring the shielding, the contribution of the coherent backscattering can artificially increase (depending on the cluster's structure and its absorption properties). To illustrate this speculation, we present the polarization phase function obtained for randomly oriented clusters composed of 15 spheres in contact with size parameters ranging from approximately 1.0 to 1.6 . We chose this range because the aggregates consisting of such monomers most often (depending on the refractive index and structure) show the negative branch of polarization (e.g., Petrova et al., 2000; 2004 and references therein). The clusters were generated by the ballistic particle cluster aggregation method (Meakin 1983). To calculate the light scattering characteristics, we used the code based on the superposition T-matrix method (Mackowski and Mishchenko, 1996). To ignore the near-field components, we introduced the corresponding changes into the computation code (Tishkovets, 2008).

Let us consider the clusters slightly differing in the packing density of particles. The porosity of an aggregate particle was determined using the concept of the characteristic radius $R_{\mathrm{c}}=(5 / 3)^{1 / 2} \times R_{\mathrm{g}}$ (Kozasa et al., $1992)$, where $R_{\mathrm{g}}$ is the gyration radius of the aggregate. Then, the porosity is $\rho=1-N \times\left(r_{\text {aver }} / R_{\mathrm{c}}\right)^{3}$, where $r_{\text {aver }}$ is the average radius of the composing particles and $N$ is their number. Figure 6 shows the polarization in the backscattering domain for the clusters with $\rho=0.52$ 


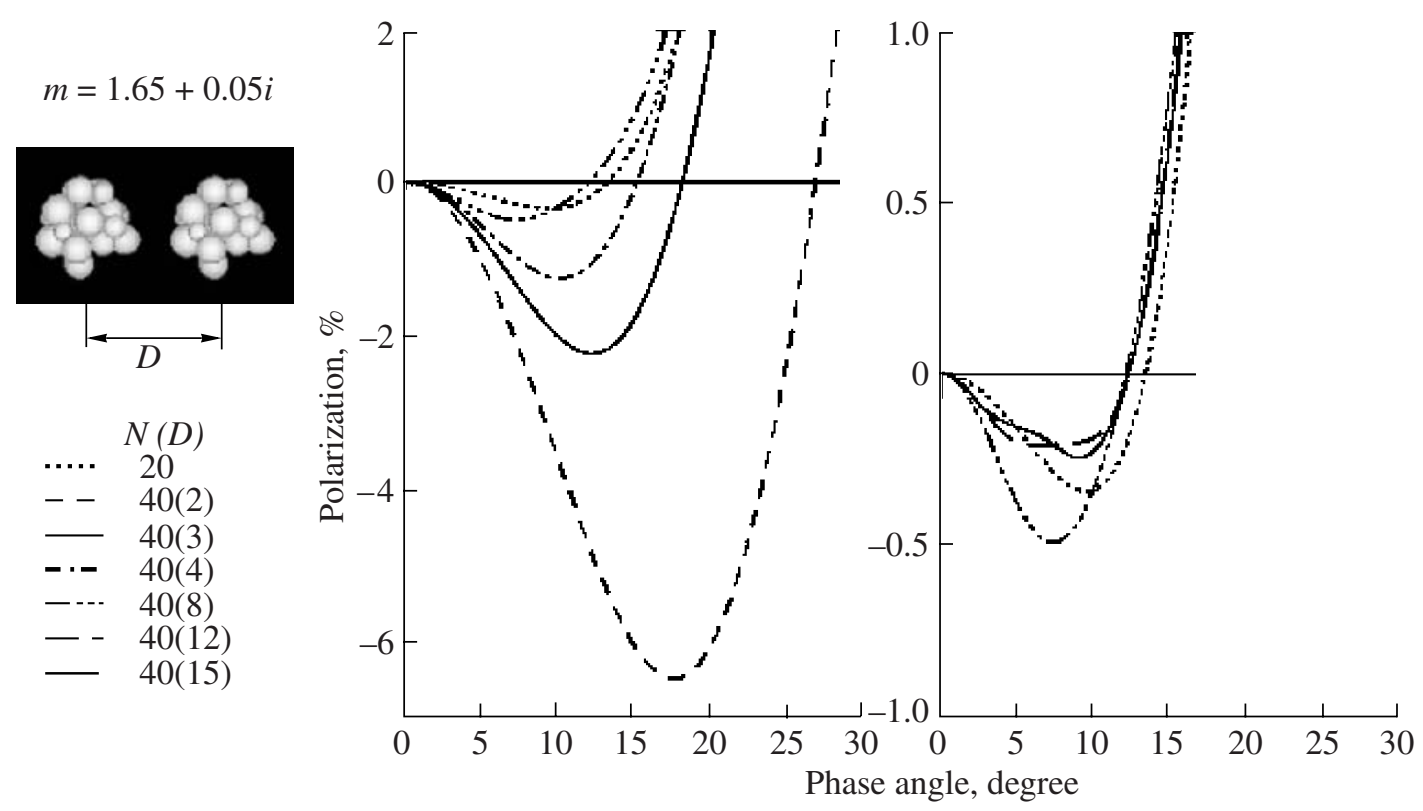

Fig. 7. The phase dependence of the degree of linear polarization in the backscattering domain is plotted for a randomly oriented cluster of 20 particles each (dotted curves) and a system of such two clusters at distances $D=2,3,4$, and $8 X_{m}($ left) and $D=8,12$, and $15 X_{m}$ (right). The refractive index is given in the figure.

and 0.61. For the more compact cluster (Fig. 6a), where the interaction of particles in the near field should be more effective, the negative branch is more pronounced. Moreover, in this cluster the mutual shielding of particles in the near field likely plays a more important role than that in the fluffy cluster (Fig. 6b). This is evident from the fact that, for the compact cluster, the model ignoring the near field produces the strengthening of the negative branch with respect to the normal model. In other words, when the shielding is ignored, the coherent backscattering contribution increases, since more particles come to be involved to the multiple scattering process. The fluffier cluster produces the weak negative branch also probably due to the field inhomogeneity near the particles, and the conditions favorable for the coherent backscattering activity are absent, since the ignoring of the near-field components leads to the disappearance of the negative branch of polarization. This example shows that such a trick as "turning on" and "turning off" the near-field components in the complex ensembles allows no distinguishing between the effects caused by different mechanisms.

A more helpful instrument for comparing the efficiencies of the interaction mechanisms in the near field and the coherent backscattering is the ensembles of clusters, the packing density of which can be varied by changing the distances between the clusters. Though it is impossible to calculate the characteristics of very large systems that could serve as models of the real regolith because the capabilities of the computers avail- able are limited, the analysis of the scattering characteristics of even small groups of clusters allows useful conclusions to be made. For this purpose, we generated the clusters composed of 20 polydisperse particles analogous to those considered above (see the insert in Fig. 7).

We calculated the scattering characteristics of the ensembles consisting of two such clusters, varying the distance $D$ between them. The polarization phase curves of the individual cluster and a pair of the clusters are shown in Fig. 7 for the backscattering domain. The individual cluster produces the weak negative branch of polarization that becomes much more prominent if the second cluster is placed next to the first one. The polarization minimum and the inversion angle move to larger phase angles. If we separate the clusters and move them away from each other the near-field effect weakens and the relative contribution of coherent backscattering increases, which is indicated by the shift of the polarization minimum to opposition. Note that the coherent backscattering mechanism should produce a narrow minimum of polarization in the immediate vicinity of the zero phase angle. At $D=8 X_{m}$ (where $X_{m}$ is the size parameter of the cluster obtained from the maximal geometric cross-section), the negative branch becomes almost symmetric, and at $D=12 X_{m}$ the coherent backscattering even causes an additional bump in the negative branch of polarization. If the separation between the clusters increases further, the multiple scattering between the clusters becomes negligible and the nega- 

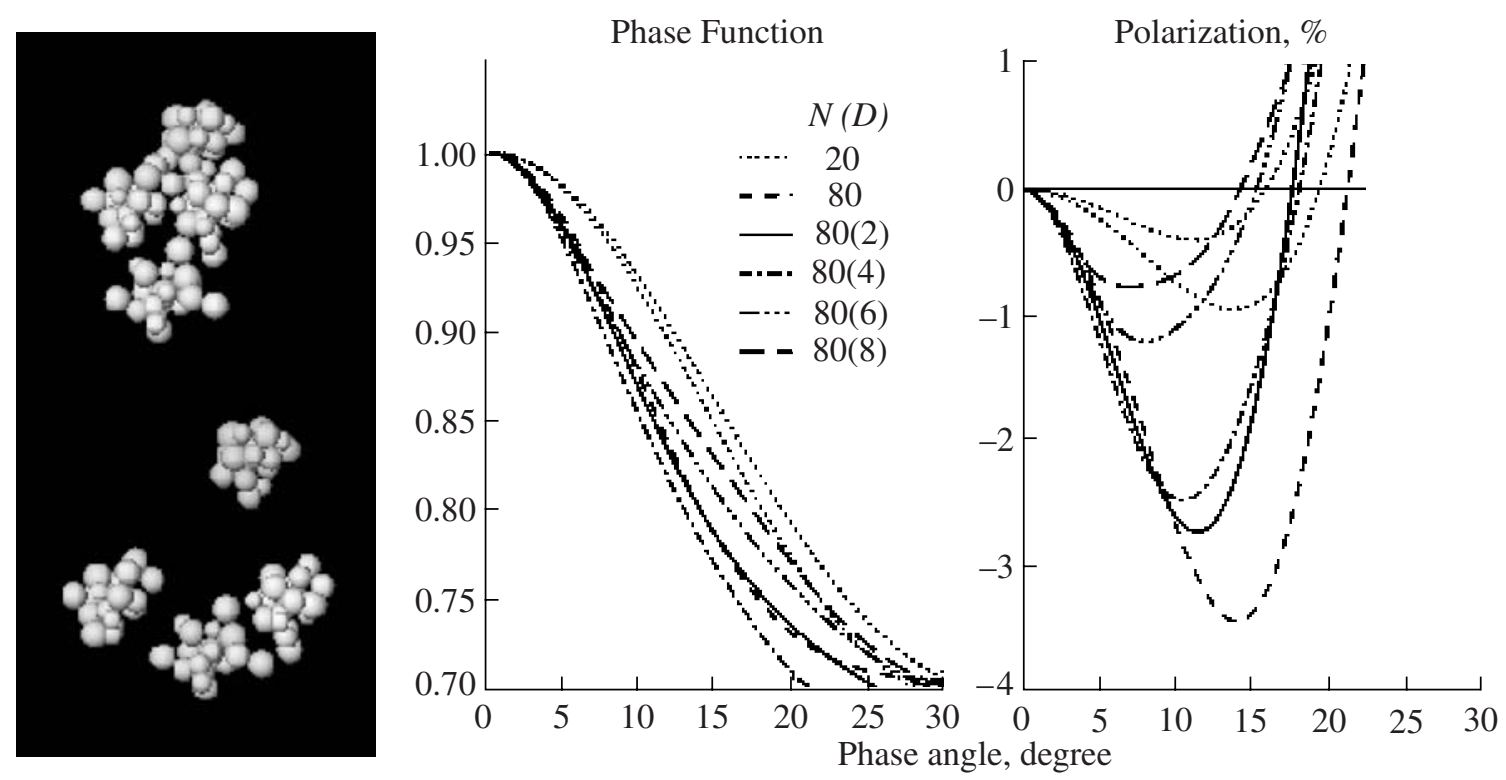

Fig. 8. In the left image, there is a perspective view of two configurations consisting of four clusters separated by distances $D=2 X_{m}$ (upward) and $4 X_{m}$, respectively (see the text). The phase dependences of the intensity (in the center, normalized to 1 at opposition) and the degree of linear polarization (right) in the backscattering domain are plotted for an ensemble of four randomly oriented different clusters of 20 particles each (the models for two of them are shown with dotted curves) at distances $D=2,3,4$, and $8 X_{m}$. The model for a compact cluster of 80 particles is shown with a short-dash curve. The refractive index is the same as that used for the models in Fig. 7.

tive branch of the ensemble approaches that of the individual clusters.

To examine this problem for more complex ensembles, we generated clusters consisting of 20 polydisperse particles and slightly differing in structure; we put them at the corners of a slightly deformed tetrahedron. Each cluster touched at least one other cluster at least at one point. Then the clusters were moved away from each other, preserving the initial "lattice." Examples of configurations of four clusters at distances $D=2 X_{m}$ and $4 X_{m}$, as well as the results of the model calculations for the analogous configurations and for the compact aggregate composed of 80 particles of the same sizes as those in the 20-particle clusters are shown in Fig. 8. It is seen that for this compact aggregate of 80 particles, the negative branch of polarization is substantially deeper and wider than for the sparser ensemble composed of four clusters in contact. Evidently, this is a consequence of the higher effectiveness of the wave inhomogeneity near the particles in the compact aggregate. This effect becomes less important for the sparser aggregate (a configuration of four clusters in contact), and even less important when the clusters are moved away from each other. The effectiveness of the coherent backscattering mechanism behaves in the opposite way: when $D$ is increasing, the polarization minimum is moving toward opposition. It is interesting to note that at the same time there is a change in the way the phase curve of intensity approaches opposition. Due to the confined geometry of scattering in small compact structures, the phase curves always have a rather large radius of curvature close to opposition (Etemad et al., 1987; Mishchenko, 1996). If the size of the scattering ensemble increases, this radius decreases, and the opposition peak of brightness becomes sharper. They are, however, not as steep as those measured in the particulate samples (see, e.g., Nelson et al., 2000; Shkuratov et al., 2002).

One may evidently expect that the effect shown in Fig. 8 should be more pronounced for less dense ensembles composed of a larger number of clusters, where the coherent backscattering mechanism plays a more important role. To some extent, this is confirmed by the models calculated for configurations consisting of three, four, and seven clusters of 20 particles each (Fig. 9). For all these models, $D=8 X_{m}$. For the model for seven clusters, two bumps are seen in the negative branch of polarization: at a phase angle of $5.5^{\circ}$ and at about $11^{\circ}$. Although the minimum at $5.5^{\circ}$ is not as deep and as close to the opposition as that measured in highalbedo celestial objects (e.g., Rosenbush et al., 2002), we may expect that more complicated larger sparse ensembles will produce model phase curves more similar to those observed. Furthermore, it is seen in Fig. 9 that when the ensemble becomes larger (as well as when the distance between the clusters grows (Fig. 8)), the curvature of the intensity curve near opposition becomes narrower. 


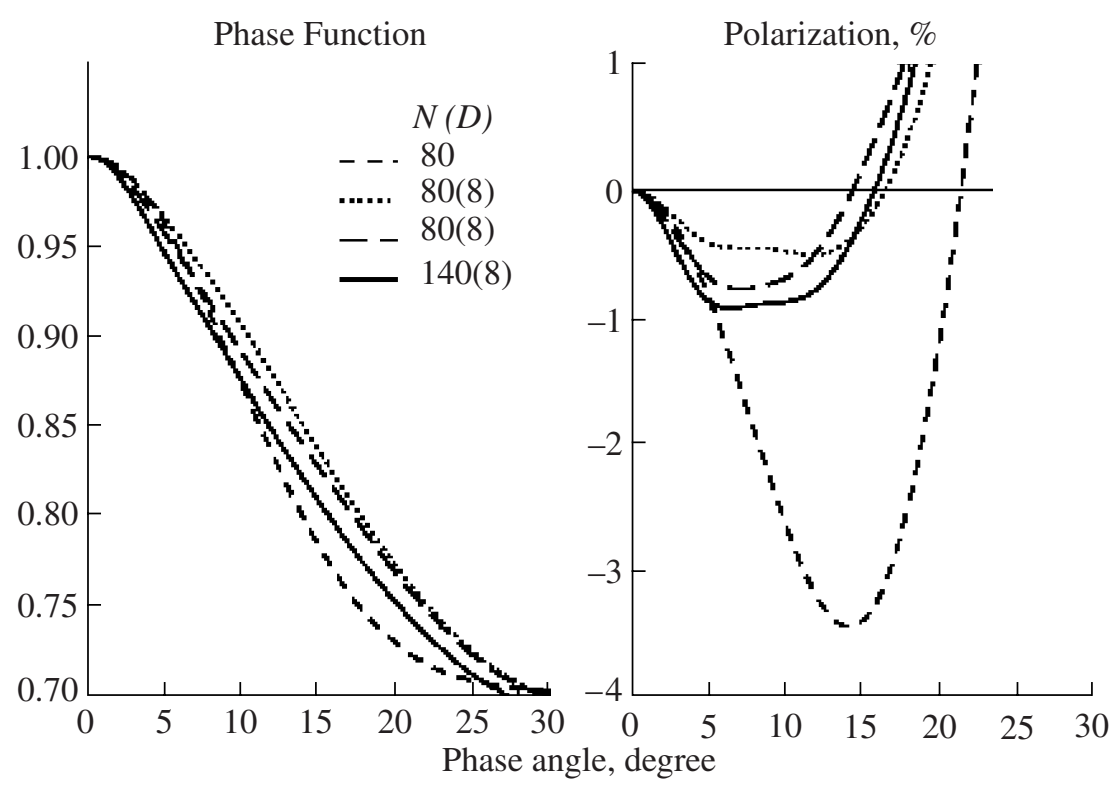

Fig. 9. The backscattering domain of the phase dependence of the intensity (left, normalized to 1 at opposition) and the degree of linear polarization (right) for an ensemble consisting of 3, 4, and 7 randomly oriented different clusters of 20 particles, each at a distance $D$ from each other. The model for a compact cluster of 80 particles is shown with a short-dash curve. The refractive index is the same as that used for the models in Fig. 7.

Both these effects-the shift of the polarization minimum to opposition (or, even appearance of a second bump or minimum) and the decrease of the curvature of the intensity phase curve near opposition-indicate that the contribution of the coherent backscattering mechanism increases with respect to that of the nearfield effect when the distances between the scatterers grow. However, the confined geometry of the ensembles modeled does not allow us to create curves with a deep polarization minimum and a sharp enhancement of intensity near opposition like those observed. The investigation of more branchy configurations turned out to be beyond the capabilities of the available computer. However, our purpose is to show the tendency provided by the different scattering mechanisms rather than to fit measured phase curves.

While discussing the results shown in Figs. 8 and 9, it is worth mentioning that the scattering characteristics calculated for different randomly generated individual clusters composed of polydisperse particles may vary. This is caused by the limited number of composing particles, which leads to the differences in structure of the outer layers of clusters, which, in turn, has a substantial influence on the behavior of the negative branch of polarization (Tishkovets et al., 2004b). However, because of the choice of the size range of composing particles and the refractive index, all the clusters considered here, even small, show the negative branch of polarization. We have modeled many cases of ensembles composed of 15- and 20-particle clusters. Despite individual differences, the tendency shown in Figs. 8 and 9 is conserved.

\section{OPPOSITION EFFECTS: THE RESULT OF THE MANIFESTATION OF DIFFERENT SCATTERING MECHANISMS}

The presented modeling of the scattering properties of ensembles of clusters of different porosity has shown that in the backscattering domain the phase dependence of intensity and polarization of light scattered by complex structures is mainly caused by two scattering mechanisms: on one hand, the constructive interference of waves propagating through the medium along the same pathway in direct and reverse directions (the coherent backscattering) and, on the other hand, the inhomogeneity of waves in the immediate vicinity of the particles and their mutual shielding (the near-field effect). The first mechanism works more effectively in sparse ensembles, while the second one manifests itself in more compact structures at distances comparable to the wavelength.

As has been already pointed out in the Introduction, the coherent backscattering mechanism together with the shadow-hiding mechanism is successfully used to explain the opposition peak in brightness. It has been shown that it is important even for dark surfaces like, for example, the lunar samples, where the contribution of multiple scattering is expected to be low (Hapke et al., 1998; Nelson et al., 1998). However, the whole spectrum of the opposition phenomena cannot be described 


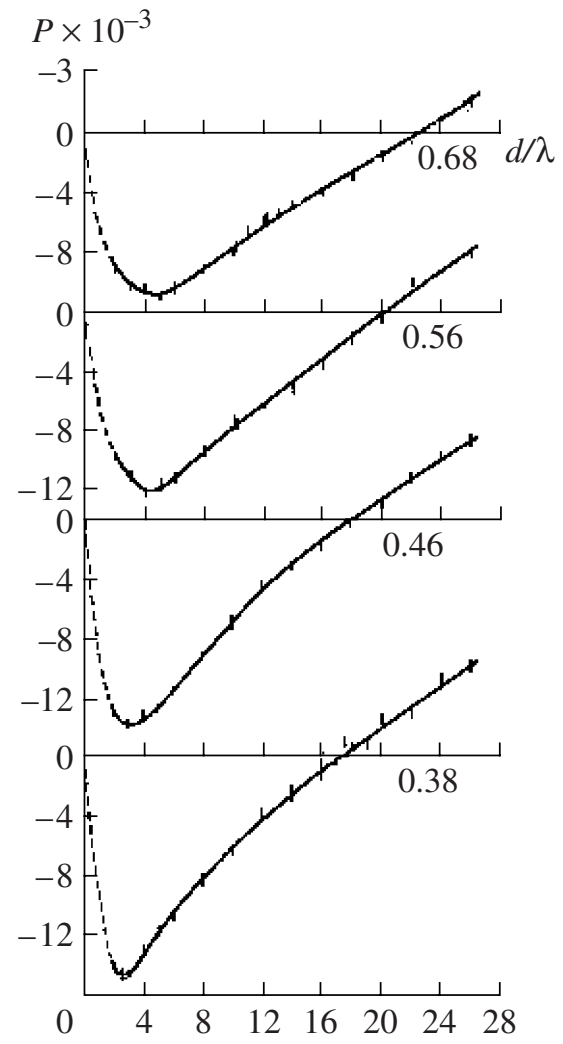

Fig. 10. The degree of linear polarization versus phase angle for alumina powders of $0.3 \mathrm{~m}$ size measured in four wavelengths. The ratio of the particle size $d$ to the wavelength $\lambda$ is indicated for each curve (adapted from the paper by Geake and Geake (1990))

only by the action of these mechanisms. Moreover, the shadow mechanism is inefficient in providing the negative branch of polarization (e.g., Shkuratov et al., 1994; Shkuratov and Grynko 2005), which often accompanies the brightness peak at opposition. Thus, the nearfield effect exactly provides the missing link that can help (together with the coherent mechanism) to explain the negative branch of polarization observed in astronomical objects and laboratory samples.

One of the examples, which cannot be explained solely by the coherent backscattering mechanism, is provided by polarimetric measurements of alumina powder (with $0.3-\mu \mathrm{m}$ grains) carried out in four wavelengths (ranging from 0.44 to $0.79 \mu \mathrm{m}$ ) by Geake and Geake (1990) (Fig. 10). Despite of their age and of the availability of numerous more modern experimental results, the measurements by Geake and Geake (1990) are of great advantage because they were made in wide spectral and angular ranges with no changes in particle size and sample porosity.

These measurements show that a deep minimum in polarization, resembling in shape that expected from the coherent mechanism, becomes wider and moves

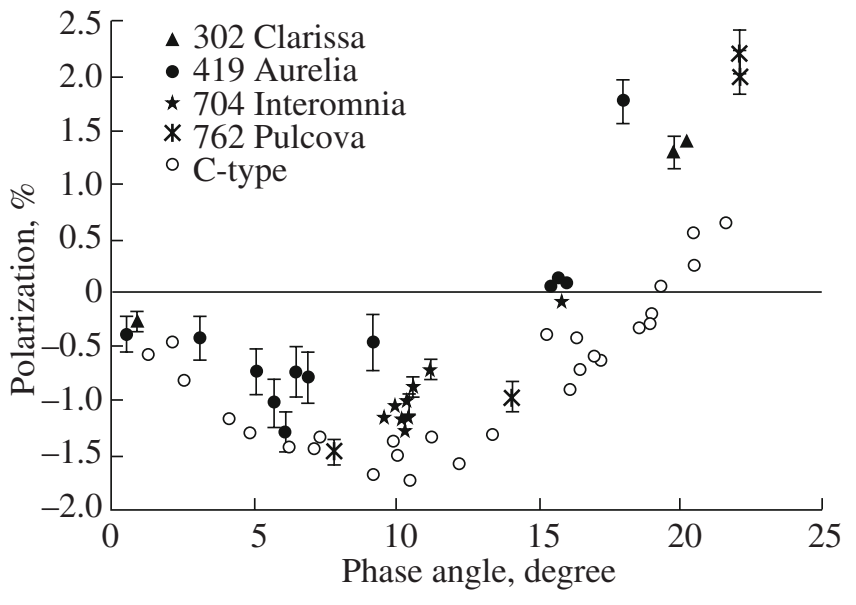

Fig. 11. The phase dependence of the degree of linear polarization for F-type asteroids measured in the V filter (adapted from the paper by Belskaya et al. (2005)).

away from opposition with decreasing wavelength. However, the interference character of the coherent mechanism requires the spectral behavior of the negative branch of polarization opposite to that observed: the half-width at half-maximum of the opposition surge due to interference, which also characterizes the position of the polarization minimum caused by this mechanism, is proportional to $\lambda /\langle l\rangle$, where $\langle l\rangle$ is the mean distance between the start and final particles in the way of the light scattered in the medium (Mishchenko et al., 2006b). It is worth noting that the special parameters introduced into the formulas describing the coherent backscattering characteristics in order to adapt them to the densely packed media allow the increase of the halfwidth of the opposition surge with decreasing wavelength to be obtained at small ratios of the particle sizes to the wavelength (Mishchenko, 1992). Nevertheless, we believe that accounting for the near field provides a more natural explanation of the spectral behavior of the negative branch of polarization observed in this experiment. If $\lambda \gg d$, (the lower plot in Fig. 10), the field inhomogeneity in the vicinity of particles is insignificant and barely affects the scattering characteristics. In this case, the coherent backscattering mechanism produces a rather narrow minimum of polarization near opposition. If the wavelength is comparable to the sizes of particles (the upper plot in Fig. 10), the near-field effect makes the polarization minimum less pronounced, moves it away from opposition, and widens the negative branch of polarization.

Though the invoking of the near-field effect may help to explain why in some cases (like in the experiments by Geake and Geake (1990)) the spectral behavior of the opposition phenomena differs from that predicted by the coherent backscattering mechanism, one should have in mind that a wide size range of the parti- 
cles and a complex hierarchic structure of the upper layer of the regolith surfaces contribute to this phenomenon as well. Hence, we would like to warn the readers to conclude that the interplay of the near-field effect and coherent backscattering alone can explain why the width of the opposition peak and of the negative branch of polarization observed for many astronomical objects and laboratory samples does not depend on wavelength (e.g., Nelson et al., 2002).

Another example of observations that can be attributed to the predominant influence of the near-field effect on the scattering characteristics is the weak negative branch of polarization detected recently in dark Ftype asteroids (Fig. 11) (Belskaya et al., 2005). Such an explanation follows not only and not so much from the very low geometrical albedo (less than 5\%) and the absence of any nonlinear opposition brightening observed for these asteroids, but from the shape of the negative branch: it is asymmetric and its minimum is located far from opposition. It is rather similar to that obtained for small compact ensembles of clusters (see Figs. 8 and 9) where the coherent mechanism is inefficient and the contribution of the near-field effect dominates.

It is also worth to be mentioned here that in dark asteroids the usual correlation between the angular parameters of the opposition effects in brightness and polarization of relatively high-albedo objects was not found (e.g., Belskaya et al., 2003). This can be explained not only by the increase of the shadow hiding contribution to the opposition brightening for the objects with low albedo, but also by the strengthening in the field inhomogeneity close to the particles. It is more substantial for materials with a high refractive index and influences both the brightness and the polarization. High-albedo fluffy surfaces can be considered as optimal for the manifestation of coherent backscattering, while in the darker surfaces the shadow hiding and the near-field effect reveal themselves more pronouncedly.

We did not intend to reanalyze here the huge amount of the results obtained in astronomical observations and laboratory measurements of the opposition effects. Here, we considered especially those observational facts we know that cannot be explained only by invoking the shadow hiding and coherent backscattering mechanisms. Our purpose, in particular, is to draw attention to the near-field effect, a scattering mechanism often ignored, despite of its importance, in the analysis of the opposition effects. To distinguish quantitatively the contributions of different mechanisms in the phase functions of brightness and polarization of natural objects and even well-controlled laboratory samples seems to be hardly possible.

\section{CONCLUSIONS}

The nature of the nonlinear increase in brightness and the negative branch of polarization with decreasing a phase angles near opposition is still obscure. The shadow hiding and coherent backscattering are the mechanisms mentioned most frequently in this connection. However, there is one more scattering mechanism - the near-field effect-which is often ignored in the analysis of the opposition phenomena and which can contribute considerably to the light scattering by a densely packed medium, especially if its albedo is low. In the present study, we have first analyzed such manifestations of the near field as the field inhomogeneity in the vicinity of the scatterers and the shielding of particles by each other at distances comparable with their sizes. Then, we used the complex ensembles of varying porosity consisting of clusters as a model of the regolith and compared the contributions of the coherent backscattering mechanism and the near-field effect to the intensity and polarization of the scattered light. The modeling has shown that the phase dependences of intensity and polarization of complex structures of scatterers in the backscattering domain is mainly caused by the interplay of two scattering mechanisms: the constructive interaction of waves traveling through the medium along the same pathways but in different directions and the near-field effect caused by the inhomogeneity of waves in the immediate vicinity of particles and the mutual shielding of particles by each other. The first mechanism works more effectively in loose, sparse ensembles, while the second one noticeably manifests itself in more compact structures of particles comparable to the wavelength in size. The near-field effect seems to be the missing link that can help (together with the coherent mechanism) to interpret the phase curves of polarization observed in various laboratory samples of astronomical objects, especially in those with moderate and low albedo.

\section{ACKNOWLEDGEMENTS}

The authors are grateful to the reviewer for the constructive remarks. The work by E.V.P. was supported in part by the Russian Foundation for Basic Research (project no. 07-02-00031a).

\section{REFERENCES}

Akkermans, E., Wolf, P.E., and Maynard, R., Coherent Backscattering of Light by Disordered Media: Analysis of the Peak Line Shape, Phys. Rev. Lett., 1986, vol. 56, pp. 1471-1474.

Akkermans, E., Wolf, P.E., Maynard, R., and Maret, G., Theoretical Study of the Coherent Backscattering of Light by Disordered Media, J. Phys. (Paris), 1988, vol. 49, pp. 77-98.

SOLAR SYSTEM RESEARCH Vol. $43 \quad$ No. 22009 
Barabanenkov, Yu.N., Kravtsov, Yu.A., Ozrin, V.D., and Saichev, A.I., Enhanced Backscattering in Optics, Progr. Opt., 1991, vol. 29, pp. 65-197.

Belskaya, I.N. and Shevchenko, V.G., Opposition Effect of Asteroids, Icarus, 2000, vol. 147, pp. 94-105.

Belskaya, I.N., Shevchenko, V.G., Kiselev, N.N., et al., Opposition Polarimetry and Photometry of S- and E-Type Asteroids, Icarus, 2003, vol. 166, pp. 276-284.

Belskaya, I.N., Shkuratov Yu.G., Efimov, Yu.S., et al., The F-Type Asteroids with Small Inversion Angles of Polarization, Icarus, 2005, vol. 178, pp. 213-221.

Boehnhardt, H., Bagnulo, S., Muinonen, K., et al., Surface Characterization of 28978 Ixion (2001 KX76), Astron. Astrophys., 2004, vol. 415, pp. L21-L25, doi: 10.1051/ 0004-6361:20040005

Dollfus, A. and Bowell, E., Polarimetric Properties of the Lunar Surface and Its Interpretation. Part I. Telescopic Observations, Astron. Astrophys., 1971, vol. 10, pp. 29-53.

Dollfus, A. and Auriere, M., Optical Polarimetry of Planet Mercury, Icarus, 1974, vol. 23, pp. 465-482.

Dollfus, A. and Geake, J.E., Polarimetric and Photometric Studies of Lunar Samples, Phil. Trans. R. Soc. Lond., 1977, vol. 285, pp. 397-402.

Dollfus, A., Polarimetry of Grains in the Coma of P/Halley. II. Interpretation, Astron. Astrophys., 1989, vol. 213, pp. 469-478.

Etemad, S., Thompson, R., Andrejco, W.J., John, S., and MacKintosh, F.C., Weak Localization of Photons: Termination of Coherent Random Walks by Absorption and Confined Geometry, Phys. Rev. Lett., 1987, vol. 59, pp. 1420-1423.

Geake, J.E. and Geake, M., A Remote-Sensing Method for Sub-Wavelength Grains on Planetary Surfaces by Optical Polarimetry, Mon. Not. R. Astr. Soc., 1990, vol. 245, pp. 46-55.

Greffet, J.-J. and Carminati R., Image Formation in NearField Optics, Prog. Surface Sci., 1998, vol. 56, pp. 133237.Gradshtain, S. and Ryzhik, I.M., Tables of Integrals, Series, and Products, New York: Academic Press, 1980.

Hapke, B., Biderectional Reflectance Spectroscopy: 4. The Extinction Coefficient and the Opposition Effect, Icarus, 1986, vol. 67, pp. 264-280.

Hapke, B., Nelson, R., and Smythe, W., The Opposition Effect of the Moon: Coherent Backscatter and Shadow Hiding, Icarus, 1998, vol. 133, pp. 89-97.

Jockers, K., Observations of Scattered Light From Cometary Dust and Their Interpretation, Earth Moon Planets, 1999, vol. 79, pp. 221-245.

Kosaza, T., Blum, J., Okamoto, H., and Mukai, T., 1993. Optical Properties of Dust Aggregates. 2. Angular Dependence of Scattered Light, Astron. Astrophys., vol. 276, pp. 278-288.

Kiselev, N.N., Rosenbush, V.K., Petrova, E.V., and Jockers, K., Asteroids and Comets: A Comparison of Polarization Properties, Mem. Soc. Astron. Ital., 2002, vol. 73, no. 3, pp. 703-715.

SOLAR SYSTEM RESEARCH Vol. $43 \quad$ No. 2009
Kuga, Y. and Ishumaru, A., Retroreflectance from a Dense Distribution of Spherical Particles. J. Opt. Soc. Am., 1984, vol. A 1, pp. 831-835.

Leinert, C., Zodiacal Light - A Measure of the Interplanetary Environment, Space Sci. Rev., 1975, vol. 18, pp. 281-339.

Lyot, B., Research of the Polarization of Light from Planets and from Some Terrestrial Substances, Ann. Obs. Meudon, 1929, vol. 8, no. 1. [NASA Technical translation TT F-187 (Engl.Transl), Washington DC (1964)]

Lumme, K. and Bowell, E., Radiative Transfer in the Surfaces of Atmosphereless Bodies. I. Theory, Astron. J., 1981, vol. 86, pp. 1694-1704.

Mackowski, D.W. and Mishchenko, M.I., Calculation of the T-Matrix and the Scattering Matrix for Ensembles of Spheres, J. Opt. Soc. Am., 1996, vol. A/13, no. 11, pp. 2266-2278.

Meakin, P., The Vold-Sutherland and Eden Models for Cluster Formation, J. Colloid. Interface. Sci., 1983, vol. 96, pp. 415-424.

Mishchenko, M.I., The Angular Width of the Coherent Backscatter Opposition Effect: An Application to Icy Outer Planet Satellites, Astrophys. Space Sci., 1992, vol. 194, pp. 327-333.

Mishchenko, M.I., On the Nature of the Opposition Polarization Effect Exhibited by Saturn's Rings, Astrophys. J., 1993, vol. 411, pp. 351-361.

Mishchenko, M.I., Coherent Backscattering by Two-Sphere clusters, Opt. Let., 1996, vol. 21, pp. 623-625.

Mishchenko, M.I., Multiple Scattering, Radiative Transfer, and Weak Localization in Discrete Random Media: Unified Microphysical Approach, Rev. Geophys., 2008, vol. 46, p. RG203.

Mishchenko, M.I., Luck, J.-M., and Nieuwenhuizen, T.M., Full Angular Profile of the Coherent Polarization Opposition Effect, J. Opt. Soc. Am., 2000, vol. A/17(5), pp. 888-891.

Mishchenko, M., Travis, L.D., and Lacis, A.A., Scattering, Absorption, and Emission of Light by Small Particles, Cambridge: Cambridge Univ. Press. 2002.

Mishchenko, M.I., Rosenbush, V.K., and Kiselev, N.N., Weak Localization of Electronic Waves and Opposition Phenomena Exhibited by High-Albedo Atmosphereless Solar System Objects, Appl. Opt., 2006a, vol. 45, pp. 4459-4463.

Mishchenko, M., Travis, L.D., and Lacis, A.A., Multiple Scattering of Light by Particles. Radiative Transfer and Coherent Backscattering, Cambridge: Cambridge Univ. Press. 2006b.

Muinonen, K., Light Scattering by Inhomogeneous Media: Backward Enhancement and Reversal of Linear Polarization, PhD dissertation, University of Helsinki, 1990.

Muinonen, K., Piironen, J., Shkuratov, Yu.G., Ovcharenko, A., and Clark, B.E., Asteroid Photometric and Polarimetric Phase Effects, in Asteroids III, Bottke, W.F., Ed., Tucson: Univ. of Arizona Press, 2002, pp. 123-138. 
Muinonen, K., Coherent Backscattering of Light by Complex Random Media of Spherical Scatterers: Numerical Solution, Waves in Random Media, 2004, vol. 14, no. 3, pp. 365-388.

Nelson, R.M., Hapke, B.W., Smythe, W.D., and Horn, L.J., Phase Curves of Selected Particulate Materials: The Contribution of Coherent Backscattering on the Opposition Surge, Icarus, 1998, vol. 131, pp. 223-230.

Nelson, R.M., Hapke, B.W., Smythe, W.D., and Spilker, L.J., The Opposition Effect in Simulated Planetary Regoliths. Reflectance and Circular Polarization Ratio Changes at Small Phase Angles, Icarus, 2000, vol. 147, pp. 545-558.

Nelson, R.M., Smythe, W.D., Hapke, B.W., and Hale, A.S., Low Phase Angle Laboratory Studies of the Opposition Effect: Search for Wavelength Dependence, Planet. Space Sci. 2002, vol. 50, pp. 849-856.

Petrova, E.V., Jockers, K., and Kiselev, N.N., Light Scattering by Aggregates with Sizes Comparable to the Wavelength: An Application to Cometary Dust, Icarus, 2000, vol. 148, pp. 526-536.

Petrova, E.V., Tishkovets, V.P., and Jockers, K., Polarization of Light Scattered by Solar System Bodies and the Aggregate Model of Dust Particles, Astron. Vestn., 2004, vol. 38, no. 4, pp. 309-324 [Sol. Syst. Res. (Engl.Transl.), 2004, vol. 38 no. 4, pp. 354-371].

Petrova, E.V., Tishkovets, V.P., and Jockers, K., Modeling of Opposition Effect with Ensembles of Clusters: Interplay of Various Scattering Mechanisms, Icarus, 2007, vol. 188, pp. 233-245.

Petrova, E.V., Tishkovets, V.P., and Jockers, K., Rebuttal to Comment on "Modeling of Opposition Effect with Ensembles of Clusters: Interplay of Various Scattering Mechanisms" by Elena V. Petrova, Victor P. Tishkovets, Klaus Jockers 2007 [Icarus 188, 233-245], Icarus, 2008, vol. 194, pp. 853-856.

Rosenbush, V., Kiselev, N., Avramchuk, V., and Mishchenko, M., Photometric and polarimetric opposition phenomena exhibited by Solar System bodies, in: Optics of Cosmic Dust, Videen, G. and Kocifaj, M., Eds., Dordrecht: Kluwer Academic Publishers, 2002, pp. 191-224.

Rosenbush, V., Kiselev, N., and Avramchuk, V., Similarity and Diversity in Photometric and Polarimetric Opposition Effects of Small Solar System Bodies, J. Quant. Spectrosc. Rad. Trans., 2006, vol. 100, pp. 325-339.

Shkuratov, Yu.G., New Mechanism of the Negative Polarization of Light Scattered by Atmosphereless Cosmic Bodies, Astron Vestnik, 1989, vol. 23, pp. 176-180.

Shkuratov, Yu.G., Muinonen, K., Bowell, E., et al., A Critical Review of Theoretical Models of Negatively Polarized Light Scattered by Atmosphereless Solar System Bodies, Earth Moon Planets, 1994, vol. 65, pp. 210-246.

Shkuratov, Yu., Ovcharenko, A., Zubko, et al., The Opposition Effect and Negative Polarization of Structural Analogs for Planetary Regoliths, Icarus, 2002, vol. 159, pp. 396-416.

Shkuratov, Yu., Ovcharenko, A., Zubko, E., et al., The Negative Polarization of Light Scattered from Particulate Sur- faces and of Independently Scattering Particles, J. Quant. Spectrosc. Rad. Trans., 2004, vol. 88, pp. 267-284.

Shkuratov, Yu.G. and Grynko, Ye.S., Light Scattering by Media Composed of Semitransparent Particles of Different Shapes in Ray Optics Approximation: Consequences for Spectroscopy, Photometry, and Polarimetry of Planetary Regoliths, Icarus, 2005, vol. 173, pp. 16-28.

Shkuratov, Yu., Bondarenko, S., Ovcharenko, A., et al., Comparative Studies of the Reflectance and Degree of Linear Polarization of Particulate Surfaces and of Independently Scattering Particles, J. Quant. Spectrosc. Rad. Trans., 2006, vol. 100, pp. 340-358.

Stephen, M.J. and Cwilich, G., Rayleigh Scattering and Weak Localization: Effects of Polarization, Phys. Rev., 1986, vol. B34, pp. 7564-7572.

Tishkovets, V.P., Backscattering of Light by Close-Packed System of Particles, Opt. Spectrosc., 1998, vol. 85, pp. 212-217.

Tishkovets, V.P. and Litvinov, P.V., Opposition Effects in Light Scattering by Regolith-Type Media, Astron. Vestn., 1999, vol. 33, no. 2, pp. 186-192 [Sol. Syst. Res. (Engl.Transl.), 1999, vol. 33, no. 2, pp. 162-168.

Tishkovets, V.P., Shkuratov, Yu.G., and Litvinov, P.V., Comparison of Collective Effects at Scattering by Randomly Oriented Cluster of Spherical Particles, J. Quant. Spectrosc. Rad. Trans., 1999, vol. 61, pp. 767-773.

Tishkovets, V.P., Litvinov, P.V., and Tishkovets, S.V., Interference Effects in Backscattering of Light by aLayer of a Discrete Random Medium, Opt. Spectrosc., 2002a, vol. 93, pp. 899-907.

Tishkovets V.P., Litvinov P.V., and Lyubchenko M.I., Coherent Opposition Effects for Semi-Infinite Discrete Random Medium in the Double-Scattering Approximation, J. Quant. Spectrosc. Rad. Trans., 2002b, vol. 72, pp. 803-811.

Tishkovets V.P. and Mishchenko, M.I., Coherent Backscattering of Light by a Layer of Discrete Random Medium, J. Quant. Spectrosc. Rad. Trans., 2004, vol. 86, pp. 161180.

Tishkovets, V., Litvinov, P., Petrova, E., et al., Backscattering Effects for Discrete Random Media: Theoretical Results. in: Photopolarimetry in Remote Sensing, Videen, G., Yatskiv, Y., and Mishchenko, M., Eds., Dordrecht: Kluwer Academic Publishers, 2004a, pp. 221-242.

Tishkovets, V.P., Petrova, E.V., and Jockers, K., Optical Properties olf Aggregate Particles Comparable in Size to the Wavelength, J. Quant. Spectrosc. Rad. Trans., 2004b, vol. 86, pp. 241-265.

Tishkovets, V.P. and Jockers, K., Multiple Scattering of Light by Densely Packed Random Media of Spherical Particles: Dense Media Vector Radiative Transfer Equation, J. Quant. Spectrosc. Radiat. Transfer, 2006, vol. 101, pp. 54-72.

Tishkovets, V.P., Incoherent and Coherent Backscattering of Light by a Layer of Densely Packed Random Media, J. Quant. Spectrosc. Radiat. Transfer, 2007, vol. 108, pp. 454-463. 
Tishkovets, V.P., Light Scattering by Closely Packed Clusters: Shielding of Particles by Each Other in the Near Field, J. Quant. Spectrosc. Radiat. Transfer, 2008, vol. 109, no. 16, pp. 2665-2672.

van Albada, M.P. and Lagendijk, A., Observation of Weak Localization of Light in a Random Medium, Rhys. Rev. Lett., 1985, vol. 55, pp. 2692-2695.

Varshalovich, D.A., Moskalev, A.N., and Khersonskii, V.K., Quantum Theory of Angular Momentum, Singapure: World Scientific, 1988.
Wolf, P.E. and Maret, G., Weak Localization and Coherent Backscattering of Photons in Disordered Media, Rhys. Rev. Lett., 1985, vol. 55, pp. 2696-2699.

Wolf, P.E., Maret, G., Akkermans, E., and Maynard, R., Optical Coherent Backscattering by Random Media: An Experimental Study, J. Phys. (Paris), 1988, vol. 49, pp. 63-75.

Zellner, B. and Gradie, J., Polarization of the Reflected Light of Asteroid 433 Eros, Icarus, 1976, vol. 28, pp. 117-123.

Zellner, B., Leake, M., Labertre, T., et al., The Asteroid Albedo Scale: I. Laboratory Polarimetry of Meteorites, Proc. 8th Lunar Sci. Conf., 1977, pp. 1091-1110. 